\title{
Manufacturing defect in endotracheal tube- An airway challenge
}

\author{
Pankaj Kumar Gupta ${ }^{1, *}$, Neha Shrivastava ${ }^{2}$ \\ ${ }^{\mathbf{1}}$ Associate Consultant, ${ }^{\mathbf{2}}$ Senior Resident, Dept. of Anaesthesiology and Critical Care, ${ }^{\mathbf{1}}$ Sarvodaya Hospital, Faridabad, Haryana, \\ ${ }^{2}$ ESIC Medical College, Faridabad, Haryana, India \\ *Corresponding Author: \\ Email: pankaj310787gupta@gmail.com
}

Received: $26^{\text {th }}$ November, 207

Accepted: $04^{\text {th }}$ October, 2017

\begin{abstract}
Endotracheal tube is a device commonly used in patients requiring general anaesthesia and mechanical ventilation. It becomes very important to ascertain its quality so that patient's health is not compromised and he or she is not exposed to undue risks. We hereby describe an incidence of manufacturing defect in endotracheal tube leading to compromised patient ventilation and the steps to recognize and rectify it.
\end{abstract}

Keywords: Manufacturing defect, Endotracheal tube, Air leak.

\section{Introduction}

Endotracheal tube is the most commonly used device for ventilation of patients as part of anaesthesia and intensive care unit. Any defect in tube is associated with inadequate ventilation leading to critical events like hypoxia, hypercarbia, aspiration, hemodynamic and neurologic sequelae. We present a case of defect in prepacked new endotracheal tube and highlight the need of standard monitoring and alertness to ensure adequate ventilation and prevent critical events.

\section{Case Report}

After standard induction of ASA 1 patient planned for laproscopic hernioplasty, direct laryngoscopy was done and a brand new cuffed portex endotracheal tube $8.0 \mathrm{~mm}$ of whose balloon was checked for leak was inserted, cuff inflated and mechanical ventilation started in volume control mode at settings of tidal volume $500 \mathrm{ml}$, respiratory rate $12 / \mathrm{min}$. The position of tube was confirmed with auscultation and capnogram. But as soon as ventilation was started a hissing sound of air leak was noticed coming from the mouth of the patient with each breath delivered by ventilator. Also the ventilator showed leak with a difference of approximately $75 \mathrm{ml}$ between inspired and expired tidal volume. We checked cuff pressure with the help of cuff pressure monitor, integrity of the circuit and connections, position of tube was rechecked with direct laryngoscopy to look for proximal migration of tube but despite everything being in correct position the leak persisted. Finally it was decided to change the endotracheal tube with a new tube. For this tube exchanger was used over which new endotracheal tube was placed, cuff inflated and ventilation started after confirming the position of the tube. There was no leak this time. The case was conducted uneventfully. The original endotracheal tube was checked for leak with clamp placed proximally to murphy's eye and placing it under water and ambu bag was connected to its connector. As the bag was compressed bubbles were seen rising from the tube at the point where pilot bag line enters the wall of tube but there was no leak in cuff as can be seen in figure 1 and 2. It was a case of manufacturing defect in endotracheal tube with leak in the wall of the tube.

\section{Discussion}

As part of our training as anaesthesiologist we have all been trained in routine check-up of endotracheal tube with cuff inflation and looking for gross defects in the tube. But this case emphasizes that some manufacturing defects are missed on routine check-up. This incidence emphasizes on routine preoperative evaluation of the endotracheal tube to be used, using the correct size of the tube, inserting it to proper length, proper inflation of cuff to appropriate pressure, checking adequate ventilation with newer workstations within built spirometers display both delivered and exhaled tidal volume with leak volume, peak pressure, frequency, capnography for measurement of etCO2 and shape of capnogram. These are well versed to us but sometimes are missed in our daily routine and workload. The vigilance and alertness of the attending anaesthesiologist can save a catastrophe. Various manufacturing defect in endotracheal tube have been reported by Gettelman et al, Sachdeva et al, Lewer et al and Singha et al. ${ }^{1-3}$ The commonly described defects are in the cuff, pilot line, pilot bag, inflation valve, defect in the wall of endotracheal tube, kinking of the tube, herniation of cuff causing obstruction and defective connector. With the advent of transparent tubes gross defects can be easily visualised but minor defects are missed. Anaesthesiologists (ASA) House of Delegates in their statement has suggested that a single use ETT can be opened to check for any manufacturing defect and possible cuff leak. ${ }^{4}$

Common causes of air leak can be deflated cuff, loose connections, improper placement of tube, cuff 
herniation above vocal cords, defect in tube or circuit and airway rents or fistula.

In cases where the defect is recognized after placement of endotracheal tube proper arrangement of difficult airway cart, all airway equipment should be checked then decision to replace the tube can be taken either with help of tube exchanger or if no difficulty suspected as in previous attempt after suctioning of mouth and deflation of cuff the endotracheal tube is removed and new tube is secured in placed with direct laryngoscopy. The defective tube should be checked again for the type of defect and ascertain it as the cause of air leak. The batch number and company of this tube should be noted so that extra vigilance may be kept for that particular batch and company and also company should be notified about the defect in their product so that they can improve their standards and such events are averted.

\section{Conclusion}

This case report stresses on the need to check the medical devices properly before use and be vigilant and alert while using them to identify device malfunction as early as possible and avoid clinical catastrophe.

\section{References}

1. Gettelman TA, Morris GN. Endotracheal tube failure: undetected by routine testing. Anesth Analg. 1995;81:1313.

2. Sachdeva R, Bhatia N. All that appears well is not well: A novel method to detect an unusual manufacturing defect of the endotracheal tube. Journal of Anaesthesiology, Clinical Pharmacology. 2015;31(4):562. doi:10.4103/0970-9185.169092.

3. Lewer BM, Karim Z, Henderson RS. Large air leak from an endotracheal tube due to a manufacturing defect. Anesth Analg 1997;85:944-5.

4. Singha SK, Kujur S. Unusual manufacturing defect of the endotracheal tube: Problem revisited. Indian J Crit Care Med [serial online] 2016 [cited 2017 Sep 26];20:130-1.

5. Statement on the Standard Practice for Infection Prevention and Control Instruments for Tracheal Intubation. American Society of Anesthesiology, Committee of Origin: Committee on Quality Management and Departmental Administration (QMDA). Approved by the ASA House of Delegates on October 20, 2010, and last amended on October 28, 2015. 